\title{
Kardiopulmonale Notfallsituationen mit hämatologisch-immunologischer Grunderkrankung
}

\author{
Hematologic or Immunologic Disease as Cause of Cardiopulmonary Distress
}

\section{U. Göbel \\ L. Gortner \\ T. Niehues}

Bibliografie

DOI $10.1055 / \mathrm{s}-0029-1237370$

Klin Padiatr 2009; 221: 273-274

(c) Georg Thieme Verlag KG

Stuttgart · New York

ISSN 0300-8630

Korrespondenzadresse

Prof. em. Dr. Ulrich Göbel

Heinrich-Heine-Universität

Düsseldorf

Moorenstraße 5

40225 Düsseldorf

goebel@med.uni-duesseldorf.de

\section{Prof. Dr. Ludwig Gortner}

Kliniken für Kinder- und Jugendmedizin

Universitätsklinikum des

Saarlandes

66421 Homburg/Saar

ludwig.gortner@uks.eu

\section{Prof. Dr. Tim Niehues}

Zentrum für Kinder- und

Jugendmedizin

Lutherplatz 40

47805 Krefeld

tim.niehues@helios-kliniken.de
Bei Störungen des kardiopulmonalen Systems mit drohender oder manifester Dekompensation der Kreislauffunktion sind die diagnostischen und therapeutischen Maßnahmen in den allgemein verfügbaren Reanimationsleitlinien für Früh- und Neugeborene sowie ältere Kinder genau vorgegeben [1-3], um sowohl das akut bedrohte Leben zu erhalten als auch möglichst schnell die Art der Störung zu erkennen.

Entsprechende Leitlinien auf europäischer Ebene wurden publiziert und in der Klinischen Pädiatrie gewürdigt $[8,10]$. Nach Überbrückung der akuten Notsituation ermöglicht dann die pathogenetische Abklärung die begonnene Therapie zielgerichtet fortzuführen. Gerade bei Früh- und Neugeborenen werden durch das metabolische und endokrine Screening angeborene Störungen diagnostiziert, deren schnelle und gezielte Behandlung zu einer Heilung oder dauerhaften Stabilisierung unter Fortführung der erforderlichen Therapiemaßnahmen führt. Allerdings werden nur die Erkrankungen dokumentiert und mitgeteilt, für die es wirksame Behandlungsverfahren gibt, woran sich die Interaktivität von Diagnostik und Therapie zeigt [7]. Auch wenn die genetische Diagnostik im heutigen Spannungsfeld von Wollen und Sollen somit nicht alles umsetzen darf, was sie schon kann, werden ständig Fortschritte erzielt [9].

Ziele sind eine normale Entwicklung und umfassende soziale Integration - auch unter Berücksichtigung der Handikaps - bei guter subjektiver Lebensqualität [19], selbst wenn die Rahmenbedingungen nicht immer optimal erscheinen [11]. Die meist geringe Fallzahldichte bei dem Großteil der Diagnosen erschwert den Erkenntnisgewinn durch randomisierte Studien, sodass es gezielter Unterstützungen bedarf, wie sie auf nationaler und zunehmend auch auf EU-Ebene verfügbar gemacht werden [17]. In Deutschland wirkt sich allerdings das moderne Entgeltsystem als Ressourcen schmälernd aus, das mit seinen Fallkostenpauschalen nicht auf den „besonderen Einzelfall“ ausgerichtet ist, der in der Kinder- und Jugendmedizin aufgrund der großen Zahl seltener Diagnosen alltäglich, aber immer besonders arbeitsintensiv ist [6]. Unabhängig hiervon wird aus guten Gründen entsprechend der immer feineren Diagnostik eine zunehmend differenziertere Therapie eingefordert, die dann noch häufig außerhalb der Zulassung zu verantworten ist (siehe Seiten 275-277 Roth und 312-317 Prandstetter).

Entsprechend dem medizinischen Fortschritt werden die Diagnostik- und Therapiepläne einer ständigen Adaptierung, die speziell in der Kindermedizin die altersphysiologische Besonderheiten und altersabhängige Referenzwerte zu berücksichtigen haben. Die Kombination der Untersuchungen für vital bedrohte Früh- und Neugeborene ist primär auf die Funktion von Herz und Lungen bzw. Diagnostik von Infektionen [4] ausgerichtet. Aber auch für andere Erkrankungen sind Leitlinien für die Diagnostik und Therapie allgemein verfügbar [5], sodass z.B. auch die rasche Erkennung sehr seltener Störungen endokriner Organe oder der Nieren ermöglicht wird $[13,18]$. Dagegen scheinen angeborene oder erworbene Erkrankungen der Hämatopoese oder Immunität häufiger Probleme zu bereiten, wie aktuelle Mitteilungen in der Klinischen Pädiatrie belegen. Da die wichtigsten hämatologischen Parameter zu den diagnostischen Notfallprogrammen gehören, sind für diese Schwierigkeiten besondere Gründe zu suchen. Neben der Seltenheit der Erkrankungen ist vor allem die klinische Manifestation erst jenseits der Neugeborenenperiode zu nennen, zumal dann - nach einem unauffälligen Neugeborenen-Screening - möglicherweise weniger intensiv an eine angeborene Störung gedacht wird. Weiterhin führt meist eine andere Erkrankung zur stationären Behandlung, die nicht an eine hämatologisch-immunologische Ursache denken lässt. So kann bei Säuglingen im ersten Lebenshalbjahr eine protrahiert verlaufende Gastroenteritis, bzw. die Persistenz von Rotaviren im Stuhl Ausdruck eines schweren kombinierten Immundefektes sein [12]. Beispielhaft für andere hämatologisch-immunologische Grunderkrankungen werden zwei vier Monate alten Säuglinge vorgestellt, die wegen einer schweren respiratorischen Infektion intensivpädiatrisch behandelt werden mussten.

Im ersten Fall ( siehe Seite 305-307 Hütten) führte eine schwere Pneumonie zum respiratorischen Versagen; da nach Ausheilen der Infektion und Normalisierung der respiratorischen Funktion klinisch eine zentrale Zyanose bestehen blieb und die pulsoxymetrisch gemessene Sauerstoffsättigung auch nach Gesundung nicht $85 \%$ überschritt, führten weitere Untersuchungen - speziell die Hochdruckflüssigkeitschromatografie des Hämoglobins nach vorhergehender Umwandlung 
in Methämoglobin - zu der Diagnose einer HbM-Hämoglobinopathie. Maßgeblich für die hämatologische Diagnostik war das normale Thoraxröntgenbild nach Überwindung der Pneumonie [21]. Obwohl Hämoglobin M primär keinen Krankheitswert hat, scheint im vorliegenden Fall nicht nur die klinische Beurteilung beeinflusst, sondern auch - durch die Reduktion der Sauerstoffkapazität - der Krankheitsverlauf erschwert worden zu sein. Interessanterweise ist dieses Kind wegen einer Gastroschisis in der 35. Schwangerschaftswoche durch Sectio caesarea geboren und stationär behandelt worden, ohne dass eine klinische Symptomatik imponierte, die zu dieser Zeit zur Diagnose geführt hätte.

Bei dem zweiten Fall (siehe Seite 302-304 Fremerey), einem 4 Monate alten Jungen mit einem akuten Atemnotsyndrom infolge einer Infektion mit Respiratory-Syncytial-Viren (RSV) wurde aufgrund des schweren protrahierten Krankheitsverlaufs ein Hyper-IgM-Syndrom Typ 1 diagnostiziert, dem molekularbiologisch eine Mutation im CD40L-Gen zugrunde liegt. Diagnostisch wegweisend war hier die Bestimmung der Immunglobuline mit erniedrigten IgG- und IgA-Konzentrationen bei normalem IgMWert.

Bei Hämatopoese oder Störungen der Immunität ist die ätiologische Abklärung im Neugeborenenalter erschwert oder nicht möglich, wie der Fall eines Kindes mit intrauterin erworbenem Leberversagen zeigt [16]. Bei diesem primär schwerkranken und komatösen Neugeborenen ließ sich keine Infektion nachweisen, wohl aber eine Hämochromatose der Leber bei starker Erhöhung des Ferritins sowie leichter Erniedrigung des Hämoglobins und des Fibrinogens. Diese Veränderungen wurden als Folgen einer Autoinflammation interpretiert, ohne dass jedoch weiterführende Untersuchungen eine ätiologische Klärung erbrachten. Unter einer Eisen ausschleusenden und antioxidativen Therapie mit Desferrioxamin, N-Acetylcystein, Selen, Vitamin E und Prostaglandin E1 erholte sich dieses Kind vollständig von allen Krankheitssymptomen.

Des Weiteren wird in diesem Heft über ein 17-jähriges Mädchen berichtet, das ohne erkennbare Ursache anhaltend hoch fieberte (siehe Seite 318-321 Kerzel). Initial bestehende Bauchschmerzen führten zu einem chirurgischen Eingriff, der keine Pathologie zeigte. Bei zunehmender Verschlechterung des Allgemeinzustandes wurde die Patientin während des Zeitraums von drei Wochen in insgesamt fünf verschiedenen Abteilungen zweier Krankenhäuser betreut, bevor sie in eine Kinderklinik verlegt wurde. Trotz sehr umfangreicher Diagnostik und zunächst erfolgloser Antibiotikagabe wurde über den diagnostischen Schlüsselbefund einer exzessiven Erhöhung des Ferritins im Serum $(60000 \mu \mathrm{g} / \mathrm{l})$ als Ausdruck einer schweren Autoinflammation ein hämophagozytisches Syndrom diagnostiziert und behandelt. Da die angeborenen und erworbenen hämophagozytierenden Lymphohistiozytosen potentiell akut lebensbedrohlich sind und auch bei der Behandlung anderweitig chronisch kranker Kinder auftreten können, wie z.B. Mukoviszidose [15] oder Uveitis [20], werden in der Übersichtsarbeit(siehe Seite 278-285 Janka) Symptomatologie, Diagnostik, Pathogenese und Therapie in Kenntnis der Daten des entsprechenden kooperativen, multizentrischen und prospektiven Therapieoptimierungsprotokolls dargestellt [14].

Diagnostisch wichtige Parameter zur Erkennung von hämatologisch-immunologischen Erkrankungen wie das vollständige rote Blutbild, Leukozytenzahl, Differenzialblutbild, Thrombozytenzahl, CRP und Fibrinogen stehen in jedem Notfallprogramm weit vorne, können bei pathologischer Veränderung aber oft durch überlagernde andere Erkrankungen plausibel erklärt werden, bzw. werden wie bei dem 17-jährigen Mädchen anfänglich „übersehen“. Möglicherweise wird die zunehmende Verwendung des Begriffs der Autoinflammation im klinischen Alltag bei unterschiedlichen Erkrankungen den diagnostischen Blick vermehrt in diese pathogenetische Richtung lenken und die fachlich spezialisierten Diskussionen frühzeitig aktivieren. Generell ist bei protrahiert oder schwer verlaufenden Infektionen frühzeitig eine immunologische Zusatzdiagnostik einzuleiten, wie die Messung der Immunglobuline, das zahlenmäßige Verhältnis der Lymphozytensubpopulationen bzw. der Granulozytenfunktion. Störungen der Erythropoese, die sich akut durch Transfusionen überbrücken lassen, dürfen nicht vergessen werden.

\section{Literatur}

1 AWMF online Empfehlungen und Leitlinien für Diagnostik und Therapie. Aufgaben des Neugeborenen-Notarztdienstes AWMF-Reg.- $\mathrm{Nr}$ $24 / 003$

2 AWMF online Empfehlungen und Leitlinien für Diagnostik und Therapie. Erstversorgung von Neugeborenen AWMF-Reg.-Nr 24/004

3 AWMF online Empfehlungen und Leitlinien für Diagnostik und Therapie. Therapie idiopathischer Apnoen, Bradykardien und Hypoxämien bei Frühgeborenen. AWMF-Reg.-Nr 24/013

4 AWMF online Empfehlungen und Leitlinien für Diagnostik und Therapie. Bakterielle Infektionen des Neugeborenen. AWMF-Reg.-Nr $24 / 008$

5 AWMF online Empfehlungen und Leitlinien für Diagnostik und Therapie. Pädiatrie (Kinderheilkunde und Jugendmedizin): Allgemeine Pädiatrie, Endokrinologie + Stoffwechselkrankheiten, Rheumatologie. AWMF Reg.-Gruppe 027

6 Christaras A, Schaper J, Strelow $\mathrm{H}$ et al. Auswirkungen des selbstlernenden G-DRG-Systems 2004-2006 auf die Vergütung stationärer Leistungen bei pädiatrisch-hämatologisch-onkologischen Patienten am Beispiel einer Universitätsklinik. Klin Padiatr 2006; 218: 366-378

7 Göbel $U$, Gortner L. Klinische Forschung und Behandlung in der Pädiatrie. Klin Padiatr 2008; 220: 2-4

8 Gortner $L$. Guidelines for cardiopulmonary resuscitation in preterm and term neonates. Klin Padiatr 2007; 219: 50-57

9 Haas $O A$, Bodamer $O$. Genetik in der Pädiatrie als Interaktion zwischen Klinik und Labor. Monatsschr Kinderheilkd 2008; 156: 323-329

10 Hansmann G, Humpl T, Zimmermann A et al. ILCOR's new resuscitation guidelines in preterm and term infants: critical discussion and suggestions for implementation. Klin Padiatr 2007; 219: 49

11 Heinrich J, Koletzko B. Kindergesundheit und Kinderbetreuung bei unter 3-Jährigen. Monatsschr Kinderheilkd 2008; 156: 562-568

12 Hennewig $U$, Schulz A, Adams $O$ et al. Severe combined immunodeficiency signalized by eosinophilia and lymphopenia in Rotavirus infected infants. Klin Padiatr 2007; 219: 343-347

13 Holzinger A, Riepe $F \mathrm{G}$, Krone $N$ et al. Extreme hyponatremia in an infant with congenital adrenal hypoplasia due to a novel NR0B1 (DAX1) mutation. Klin Padiatr 2008; 220: 287-290

$14 \mathrm{HLH}-2004$, Treatment protocol of the Second International HLH Study 2004.. http://www.gpoh.de

15 Jost C, Dockter G, Knöpli B et al. Scedosporium-Pneumonie und hämophagozytierende Lymphohistiozytose(HLH): Ungewöhnliche Nebenwirkungen einer Kortikoidtherapie der ABPA bei CF. Klin Padiatr 2007; 218: 107

16 Keller M, Scholl-Buergi, Sergi C et al. An unusual case of intrauterine symptomatic neonatal liver failure. Klin Padiatr 2008; 220: 32-36

17 Ohmann C. Klinische Studien in der Pädiatrie: Herausforderungen und aktuelle Entwicklungen. Klin Padiatr 2008; 220: 221-223

18 Schnakenburg C von, Frankenschmidt A, Neumann J et al. Polyhydramnion, Frühgeburtlichkeit, Dystrophie, Polyurie, Obstipation, Nephrokalzinose und renale Raumforderung: Präsentation einer klassischen Tubulopathie. Klin Padiatr 2008; 220: 24-25

19 Strassburg HM, Leimer S, Platz A et al. Long-term prognosis of former very and extremely preterm babies in adulthood in Germany. Klin Padiatr 2008; 220: 61-65

20 Winterhalter S, Niehues T. TNF $\alpha$-blocking agents or conventional immunosuppressive drugs in the therapy of children with uveitis? An evidence based approach. Klin Padiatr 2008; 220: 342-347

21 Wirbelauer J, Thomas W, Darge K. Zentrale Zyanose und Verdichtungen im Thoraxröntgenbild bei einem Säugling. Monatsschr Kinderheilkd 2007; 155: 789-792 